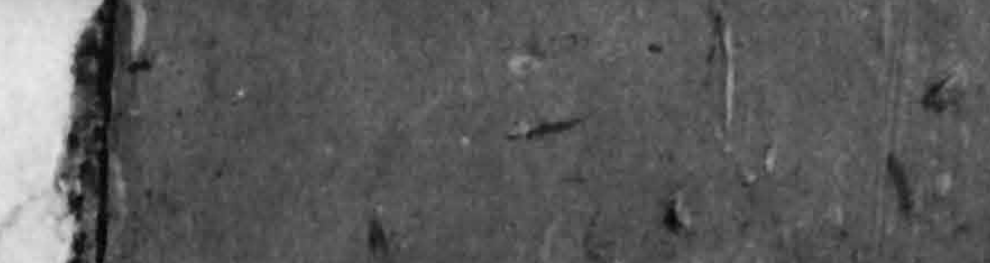

$-4$

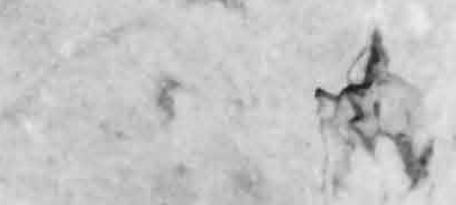

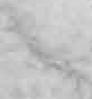
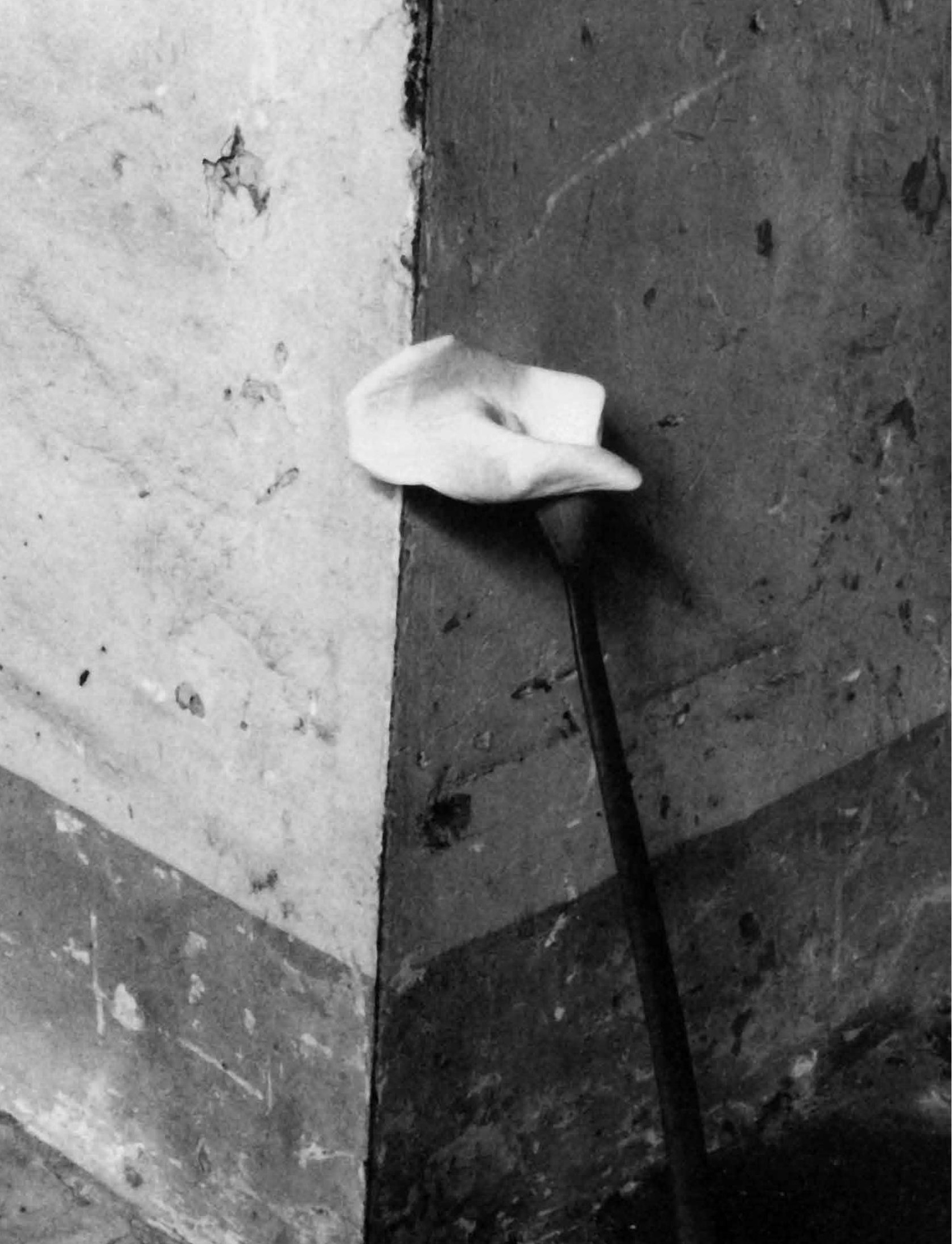


\section{Cadernos de Arquitetura e Urbanismo}

Os Cadernos de Arquitetura e Urbanismo são produzidos pelo Departamento de Arquitetura e Urbanismo da PUC Minas. Dedicam-se à divulgação de trabalhos técnico-científicos relacionados à área de arquitetura e urbanismo, especialmente os vinculados às atividades de ensino, pesquisa, pós-graduação e extensão.

Este periódico é editado anualmente desde 1993, passando à semestralidade em 2008. Desde 2010, sua edição se dá no sistema SEER - Sistema Eletrônico de Editoração de Revistas, por meio do portal do periódico, em http://periodicos.pucminas.br/index.php/arquiteturaeurbanismo.

\section{Classificação Qualis / CAPES (http://qualis.capes.gov.br/webqualis)}

B1 na área de Geografia; B2 em Arquitetura e Urbanismo e História; B3 em Sociologia e Planejamento Urbano e Regional / Demografia, e B4 em Engenharias I.

Indexadores

ICAP - Indexação Compartilhada de Artigos de Periódicos (http://www.pergamum.pucpr.br/icap/index.php)

Latindex - Sistema Regional de Información en Línea para Revistas Científicas de América Latina, el Caribe, España y Portugal (http://www.latindex.unam.mx/)

Periódico Cadastrado no CCN - Catálogo Coletivo Nacional sob o n091873-3

Editor

Antonio Grillo

Assistente editorial

Ana Flávia Veloso, Bruna Marinho Sampaio

Conselho Editorial Científico

Jeanne Marie Ferreira Freitas (PUC Minas - Presidente), Aurélio Muzzarelli (Università di Bologna / Itália), Brian Lawson (The University of Sheffield / Inglaterra), Carlos Antônio Leite Brandão (UFMG), Cláudia Damasceno (Université de Paris / França), Cláudio Listher Marques Bahia (PUC Minas), Fernando Luiz Camargos Lara (University of Michigan / EUA), Heloísa Soares de Moura Costa (UFMG), Marcio Cotrim Cunha (UFPB), Paulo Ormindo (UFBA), Ricardo Moretti (PUC Campinas), Silke Kapp (UFMG), Sônia Marques (UFRN).

Pareceristas deste volume (v. 20, n. 26 e n. 27)

Adriano Silva Fortes; Alfio Conti; Alícia Duarte Penna; Altino Barbosa Caldeira; Ana Carolina de S. Bierrenbach; Ana Claudia Duarte Cardoso; Ana Paola Oliveira Araújo; André Pereira de Souza; Angelina Dias Leão Costa; Antonio Grillo; Ayrton Portilho Bueno; Carlos Eduardo Flores de Araújo; Circe Maria Gama Monteiro; Cláudia Loureiro; Cláudia Regina Vial Ribeiro; Clécio Magalhães do Vale; Daniela Abrita Cota; Décio Rigatti; Denise Almada Horta Madsen; Denise Marques BahiaDenise Morado Nascimento; Edja Trigueiro; Eleonora Sad de Assis; Fernanda Borges de Moraes; Fernando Luiz Camargos Lara; Flávia Nacif da Costa; Flávio de Lemos Carsalade; Flávio Pereira Dias Duarte; Francisco Sales Trajano Filho; Frederico de Holanda; Geraldo Magela Costa; Glacir Teresinha Fricke; Guilherme Maciel Araújo; Iracema G e Abreu Bhering; Joana D'Arc da Silva Pinto; João Batista Santos de Assis; Joelmir Marques Silva; José Augusto Ribeiro da Silveira; Juliana Gonzaga Jayme; Juliana Maria Simões Campos; Junia Cambraia Mortimer; Katja Plotz Froiz; Luiz Cláudio Bittencourt; Manoel Teixeira Azevedo Júnior; Marcelo da Rocha Silveira; Marcelo Kraiser; Márcio Cotrim Cunha; Margarete Maria de Araújo Silva; Maria Elisa Baptista; Michele dos Anjos de Santana; Mônica Maria Cadaval Bedê; Nelci Tinem; Rachel de Castro Almeida; Rachel de Souza Vianna; Ralfo Matos; Raquel Manna Julião; Renata Moreira Marquez; Rita de Cássia Lucena Velloso; Roberta Vieira Gonçalvez de Souza; Roberto Eustáaquio dos Santos; Rodrigo Espinha Baeta; Rosana Soares Bertocco Parisi; Sandra Catharinne Pantaleão; Sandra Machado Fiuza; Simone Parella Tostes; Sônia Maria de Barros Marques;Sulamita Fonseca Lino; Tânia Maria de Araújo Ferreira; Tatiana Soledade Delfanti Melo; Willi de Barros Gonçalves

Projeto gráfico

Antonio Grillo / Adílson Cruz Júnior / José Augusto Barros

Diagramação

José Augusto Barros • www.be.net/gutobarros

Revisão

Alessandro Faleiro Marques

Endereço para correspondência

Pontifícia Universidade Católica de Minas Gerais • Departamento de Arquitetura e Urbanismo

Av. Dom José Gaspar 500 Prédio 47 • Bairro Coração Eucarístico • 30535-901 • Belo Horizonte • Minas Gerais • Brasil

E-mail: cadernos.au@pucminas.br • Tel. / Fax: (0xx31) 33194264

Política de doações e permutas

A distribuição dos Cadernos de Arquitetura e Urbanismo é feita pela Biblioteca da PUC Minas, através de doação e permuta. A política de permutas e doações garante a circulação deste periódico em mais de 15 países. Para solicitações de doação ou permuta, entrar em contato com: Pontifícia Universidade Católica de Minas Gerais • Biblioteca Padre Alberto Antoniazzi / Setor de Periódicos • Av. Dom José Gaspar, 500 Prédio $26 \bullet$ Bairro Coração Eucarístico • Belo Horizonte • Minas Gerais • Brasil •Tel/Fax: (0xx31)3319 $4175 \bullet$ e-mail: bibpe@pucminas.br

Apoio

Edição financiada pela FAPEMIG no Edital 05/2012

Versão eletrônica / submissão de trabalhos / normas para apresentação

http://periodicos.pucminas.br/index.php/arquiteturaeurbanismo 


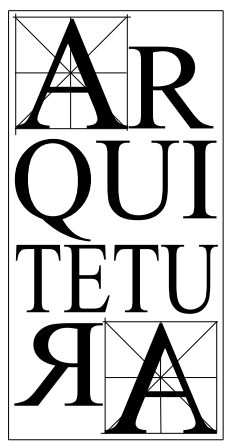

Cadernos de Arquitetura e Urbanismo

volume 20, número 27

$2^{0}$ semestre de 2013

ISSN 1413-2095

Apoio: 


\section{Pontifícia Universidade Católica de Minas Gerais}

Grão-Chanceler

Dom Walmor Oliveira de Azevedo

Reitor

Dom Joaquim Giovani Mol Guimarães

Vice-Reitora

Patrícia Bernardes

Assessor Especial da Reitoria

José Tarcísio Amorim

Chefe de Gabinete do Reitor

Paulo Roberto de Sousa

Pró-reitorias e Secretarias

Extensão - Wanderley Chieppe Felippe; Gestão Financeira - Paulo Sérgio Gontijo do Carmo; Graduação - Maria Inês Martins; Logística e Infraestrutura - Rômulo Albertini Rigueira; Pesquisa e de Pós-graduação - João Francisco de Abreu; Planejamento e Desenvolvimento Institucional - Carlos Barreto Ribas; Recursos Humanos - Sérgio Silveira martins; Arcos - Marcelo Leite Metzker; Barreiro - Renato Moreira Hadad; Betim - Eugênio Batista Leite; Contagem - Maria José Viana Marinho de Mattos; Poços de Caldas - Iran Calixto Abrão; São Gabriel - Miguel Alonso de Gouvêa Valle; Serro - Eustáquio Afonso Araújo; Guanhães - Ronaldo Rajão Santiago

\section{Departamento de Arquitetura e Urbanismo}

Diretor

Vanessa Borges Brasileiro

Colegiado

Claudio Marques Listher Bahia, Daniele Caetano Nunes de Sá, José Martins dos Santos Neto.

\section{Editora PUC Minas}

Diretor: Patrus Ananias de Souza

Coordenação editorial: Cláudia Teles de Menezes Teixeira

Assistente editorial: Maria Cristina Araújo Rabelo

Conselho editorial: Patrus Ananias de Souza (Diretor); Ângela Vaz Leão (PUC Minas); Caio César Boschi (PUC Minas); João Francisco de Abreu (PUC Minas); Maria Zilda Ferreira Cury (UFMG); Mário Neto Borges (Fapemig); Milton do Nascimento (PUC Minas); Otávio Dulci (PUC Minas); Paulo Agostinho N. Baptista (PUC Minas); Regina Helena Campos (UFMG); Sérgio de Morais Hanriot (PUC Minas)

Endereço para correspondência:

Pontifícia Universidade Católica de Minas Gerais

Rua Dom Lúcio Antunes, 180 • Coração Eucarístico • 30535-490 • Belo Horizonte • Minas Gerais • Brasil Tel: (31) $3319.9904 \bullet$ Fax: (31) $3319.9907 \bullet$ e-mail: editora@pucminas.br

C122 Cadernos de Arquitetura e Urbanismo. - v.1, n.1 (abr.. 1993- ). Belo Horizonte: Ed. PUC Minas, 1993-

v.

Semestral

ISSN 1413-2095 - versão impressa

ISSN 2316-1752 - versão eletrônica em 2003

1. Arquitetura - Periódicos. 2. Planejamento urbano - Periódicos.

I. Pontifícia Universidade Católica de Minas Gerais.

Departamento de Arquitetura e Urbanismo. 
Modernidade e moderno: temas para debater o espaço.....11 Modernity and modern: themes to discuss space Luciano Torres Tricárico

A construção de um olhar sobre a arquitetura na fotografia de Francesca Woodman (1960-1981)

Building a photographic gaze towards architecture in the works by Francesca Woodman (1960-1981) Junia Cambraia Mortimer

A imagem urbana de Liège na obra de Georges Simenon: o caso de Le pendu de Saint-Pholien

The urban image of Liège in the work of Georges

Simenon: the case of Le pendu de Saint-Pholien

Adson Cristiano Bozzi Ramatis Lima

Renata Fernandes

Impacto, risco ou vulnerabilidade: uma discussão sobre instrumentos de análise urbano-ambiental.

Impact, risk or vulnerability: a discussion on

urban-environmental analysis tools

André Luiz Prado

APP e parques lineares:

adoção de conceito ou arquétipo?

APP and linear parks: adoption of a concept, or creating archetype?

Denio Munia Benfatti

Jonathas Magalhães Pereira da Silva

Águas urbanas como elementos de estruturação do território: usos e valor na cidade contemporânea......99

Urban water on the structure of territory: uses and value in contemporary city

Sandra Catharinne Pantaleão

Contramedidas da especulação imobiliária

no mercado residencial 125

Countermeasures of speculation in the

residential market

Andreas Dittmar Weise

Jürgen W. Philips

Norberto Hochheim

Andréa Cristina Trierweiller

Antonio Cezar Bornia

Materialização de projetos: uma abordagem

metodológica

Materialization of design:a methodological approach

Rogerio Braga de Assunção

Adilson Rodrigues da Costa

Jairo José Drummond Câmara 